\title{
Introduction to special issue on Energy-Aware Simulation and Modelling (Energy-SIM)
}

It has been our pleasure to serve as guest editors of this special issue on Energy-Aware Simulation and Modelling (ENERGY-SIM) for the Sustainable Computing: Informatics and Systems (SUSCOM) journal.

The energy impact of IT infrastructures is a significant factor for many organisations. With the Natural Resources Defence Council estimating that the US data centres alone consumed 91 billion kilowatt-hours of electrical energy in 2013 - enough to power the households over New York twice-over - and estimated to grow to 139 billion kilowatt-hours by 2020 this is a significant impact on world energy usage. Furthermore, this fails to take into account all other computer resources.

There are calls for reducing computer energy consumption to bring it in line with the amount of work being performed - so-called energy proportional computing. In order to achieve this we need to understand both where the energy is being consumed within a system and how modifications to such systems will affect the functionality (such as QoS) and energy consumption of the system. Monitoring and changing a live system is often not a practical solution. There are cost implications in such work and it normally requires significant time in order to fully ascertain the long-term trends. There is also the risk that such changes could lead to detrimental impacts, either in terms of the functionality of the system or in the energy consumed. Leading to a situation where it is considered too dangerous to perform anything other than the most minor tweak to a system. Modelling and simulation provides an alternative approach to evaluating where energy is being consumed and the impacts of changes to system. It also offers the potential for much faster turn-around along with the ability to evaluate the impact of many different alternatives simultaneously.

This special issue provides the scientific community a dedicated forum for presenting new research, development challenges, developing new techniques and advanced information technology solutions in green computing through the use of Modelling and Simulation.

Aligning with the high-quality standards of SUSCOM, we were highly selective and accepted only the top contributions in this special issue after a rigorous review process. We believe that these papers reflect the current research trends and key issues related to modelling and simulation of diverse areas such as embedded systems, network links, virtual machines and data centres. The accepted papers are introduced below:

The paper "Energy-Efficient Scheduling with Reliability Guarantee in Embedded Real-Time Systems" is authored by Hongzhi $\mathrm{Xu}$, Renfa Li, Lining Zeng and Keqin Li at Hunan University and Chen Pan at Oklahoma University. The authors address the combined problems of limited energy availability and runtime faults (as a consequence of Dynamic Voltage Frequency Scaling) within embedded devices. The authors propose a greedy, energy-efficient algorithm where the processor is slowed down to a level where the task will just complete within the desired time-limit. However, if the processor encounters a fault then 
it will re-run the task at maximum frequency. Results are provided which demonstrate, through the use of simulation, that the approach is capable of providing significant improvements both in terms of energy efficiency and reliability of the embedded device.

The paper "Dual-Mode Energy Efficient Ethernet with Packet Coalescing: Analysis and Simulation" is authored by Mehrgan Mostowfi, Khalil Shafie at the University of Northern Colorado. The authors evaluate the energy impact of Dual-Mode Energy Efficient Ethernet where low power modes allow the new high-speed links to sleep during idle periods. The authors go further by demonstrate that through allowing packets to be consolidated and sent on in bursts this can significantly increase the amount of time that the network link can remain in sleep state and hence save energy. The authors present a mathematical model to support their work and go further to simulate such a network link. They argue that the delay in packet delivery can be tolerated given the reduction in energy consumption, save for those few use-cases where latency is vitally important.

The paper "An experiment-driven energy consumption model for virtual machine management systems" is authored by Mar Callau-Zori, Lavinia Samoila and Guillaume Pierre at the University of Rennes and Anne-Cécile Orgerie at CNRS. The paper addresses the problem of accurately determining the energy consumptions of VM operations such as starting a new VM or live migrating a VM. The authors propose that the configuration of the host machine (such as number of VMs) is crucial in order to build a better model of energy consumption. Through the use of this approach the authors are able to more accurately model the energy consumption than other approaches. One major benefit of their approach is the ability to model hosts which are over-provisioned, something which now happens regularly within real data centres.

The paper "Energy-proportional Profiling and Accounting in Heterogeneous Virtualized Environments" is authored by Mascha Kurpicz, Anita Sobe and Pascal Felber at the University of Neuchatela and AnneCécile Orgerie at CNRS. In this paper the authors consider the energy costs for individual Virtual Machines (VM) within a data centre. They derive a model EPAVE (Energy-Proportional Accounting in VM-based Environments) capable of computing both the proportion of static and dynamic energy cost associated with a particular VM instance. This includes PowerIndex which is able to profile energy usage on a given architecture whilst also being able to predict energy usage on a different platform. The model is used to evaluate a number of application softwares.

We hope that the readers will find the papers in this special issue informative and useful. The guest editors wish to thank the authors of all submitted manuscripts, without whom this special issue would not have been possible. They also thank the reviewers who provided a thorough evaluation of the submitted manuscripts in a timely manner. We appreciate the assistance of the Special Issues Co-Editor-in-Chief, Ishfaq Ahmad the Editor-in-Chiel, Behrooz Shirazi, and the SUSCOM editorial staff throughout the process of bringing out the special issue.

A. Stephen McGough Matthew Forshaw

School of Computing 


\section{Urban Sciences Building Newcastle University 1 Science Square Science Central Newcastle upon Tyne NE4 5TG}

Email addresses: \{stephen.mcgough,matthew.forshaw\}@newcastle.ac.uk 